\title{
Coherent nanoscale optical-phonon wave packet in graphene layers
}

\author{
I. Katayama, ${ }^{1}$ K. Sato, ${ }^{1}$ S. Koga, ${ }^{1}$ J. Takeda, ${ }^{1, *}$ S. Hishita, ${ }^{2}$ H. Fukidome,${ }^{3}$ M. Suemitsu, ${ }^{3}$ and M. Kitajima ${ }^{1,4,5, *}$ \\ ${ }^{1}$ Graduate School of Engineering, Yokohama National University, Yokohama, 240-8501 Japan \\ ${ }^{2}$ Optical and Electronic Materials Unit, National Institute for Materials Science, Tsukuba, 305-0044 Japan \\ ${ }^{3}$ Research Institute of Electrical Communication, Tohoku University, Sendai 980-8577, Japan \\ ${ }^{4}$ LxRay Co. Ltd., Nishinomiya 663-8172, Japan \\ ${ }^{5}$ Department of Applied Physics, National Defense Academy, Yokosuka 239-8686, Japan
}

(Received 9 April 2013; revised manuscript received 15 August 2013; published 4 December 2013)

\begin{abstract}
Coherent large-wave-vector phonons in graphene layers were excited by using a 7.5-fs ultrashort laser, and observed by wavelength-resolved pump-probe transient reflectivity spectroscopy. Inter-Dirac-cone electron scattering mediated by edges and defects in graphene layers drives large-wave-vector coherent $D$-mode phonons near the Dirac point ( $K$ point) of the Brillouin zone. In contrast to the normal coherent phonons generated with first-order Raman process, phonon chirp is observed, and amplitude and frequency strongly change depending on the probed wavelength. The results are discussed on the basis of doubly resonant Raman scattering process, demonstrating that generated $D$-mode coherent phonons can propagate as nanoscale optical-phonon wave packets.
\end{abstract}

DOI: 10.1103/PhysRevB.88.245406

PACS number(s): 78.67.Wj, 78.30.-j, 78.47.jg

Manipulating coherent motions of atomic or lattice vibrations is one of the keys to dynamic control of electronic and optical properties of materials. For this purpose, many studies were conducted using ultrashort laser pulses, combined with pulse-shaping techniques. ${ }^{1-4}$ However, most of the studies are limited to the excitation of $\Gamma$-point phonons in solid materials, or of localized vibrational wave packets in atomic, molecular, or self-trapped excitons. ${ }^{5,6}$ Dynamics of nanoscale phonon wave packets, information on which is indispensable for full manipulation and control of electronic and optical properties of materials, has not been investigated so far. These phonon wave packets are expected to propagate in space following the phonon dispersions in the Brillouin zone, which could provide us with a new degree of freedom in a material's control.

The propagating nanoscale wave packets are of particular interest in graphene, which is a promising candidate for nanoscale devices. Moreover, the unique conical band structure near the Fermi level of graphene ${ }^{7}$ gives rise to a strong resonant interaction between Dirac electrons and phonons, known as Kohn anomaly. ${ }^{8-10}$ The strong coupling can be useful in controlling the atomic motions through electronic excitation and vice versa. Coherent excitation of phonons with the Fermi wave vector ( $K$-point wave vector in the Brillouin zone) is a key to realize such control, which is also important for understanding the phonon dynamics and the strong electron-phonon coupling. However, ultrafast dynamics has only been investigated for the phonon modes at the $\Gamma$ point in graphene-related materials ${ }^{11-15}$ owing to momentum conservation rule reinforced in the optical measurements. If the coherent large-wave-vector phonons at the $K$ point can be excited and controlled, then the electronic states of the Dirac electrons can be directly modulated at a femtosecond timescale.

In the present study, we will demonstrate that impulsive excitation of graphene edges or defects by ultrashort laser pulses form a nanoscale optical-phonon wave packet near the $\mathrm{K}$ point in the Brillouin zone. This wave packet propagates along the graphene sheet with a group velocity of $5 \mathrm{~nm} / \mathrm{ps}$, reflecting the strong coupling between Dirac electrons and phonons. The mode is known as $D$ mode in graphene-related materials, ${ }^{16-18}$ and in contrast to the normal Raman modes at the $\Gamma$ point, originates from the doubly resonant Raman scattering process. ${ }^{19,20}$ This unique Raman process relaxes the momentum conservation rule by the electron scattering at edges or defects, enabling the excitation of coherent phonons with various large wave vectors [see the dashed arrow in Fig. 1(a)]. Because the allowed wave vectors of coherent phonons depend on the light's frequency, the broad spectrum of the ultrashort laser enhances the wave-vector bandwidth of the excited coherent phonons. A superposition of these coherent phonons with different wave vectors results in an optical-phonon wave packet whose real space displacements of carbon atoms are confined to the nanoscale as shown in Fig. 1(b). The wave packet initially localizes at the graphene edges and defects, and subsequently propagates along the graphene sheet as a coupled excitation of coherent phonons with Dirac electrons. The excitation and detection of such coherent phonons will open up a possibility of investigation of nanoscale properties of graphene without using any sophisticated space-resolved equipment such as a scanning tunneling microscope. In the following, we will show how this concept appears in the experimental results.

We performed pump-probe transient reflectivity measurements of graphene on silicon (GOS) and defective graphite (Ar-G) samples using a commercial Ti:sapphire laser (VENTEON) with a pulse duration of $7.5 \mathrm{fs}$, center wavelength of $800 \mathrm{~nm}$, repetition rate of $80 \mathrm{MHz}$, and output power of $300 \mathrm{~mW}$. The spectrum extends from 680 to $1050 \mathrm{~nm}$, making the wavelength-resolved spectroscopy meaningful. Both pump and probe beams were incident on the sample at $\sim 30^{\circ}$ from the surface's normal, and then, the anisotropic change of the reflectance due to the pump pulse was measured by the electro-optic sampling method. ${ }^{13}$ Band-pass filters with 10-nm bandwidth were placed behind the sample to observe the probe-wavelength dependence. Coherent phonon experiment with ultrashort laser pulses can detect the time-dependent frequency, amplitude and 


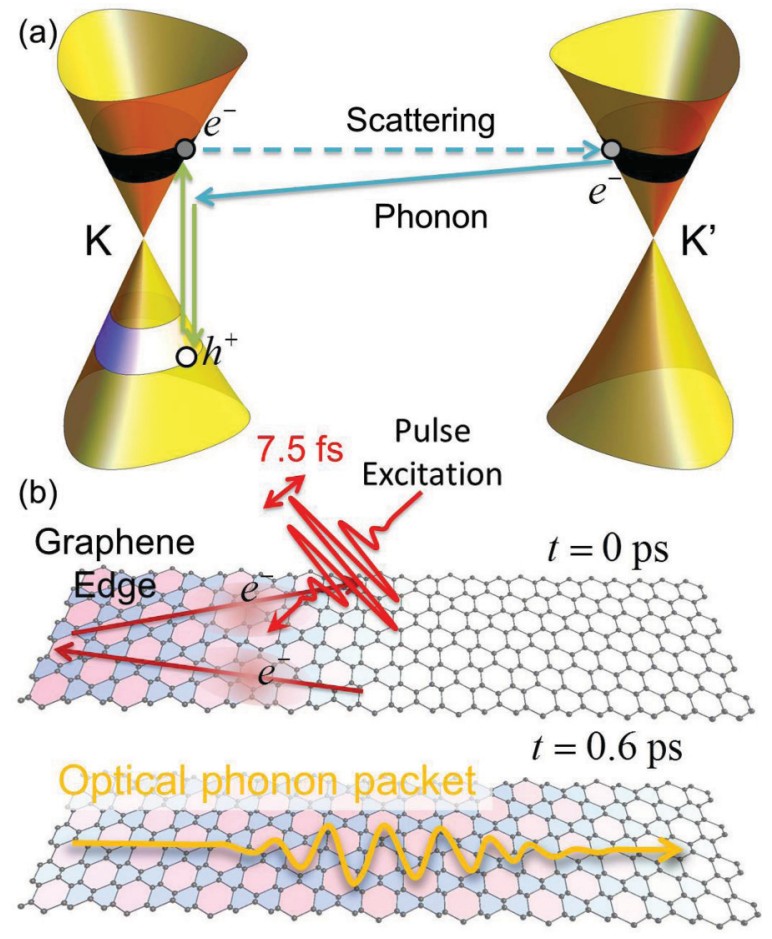

FIG. 1. (Color online) Nanoscale optical-phonon wave packets. (a) Schematic of the inter-Dirac-cone scattering that generates large-wave-vector phonons at the graphene edge. (b) The calculated nanoscale optical phonon wave packets based on the doubly resonant Raman scattering model at $t=0$ and $0.6 \mathrm{ps}$ are shown. The red and blue hexagons expand and shrink, respectively, due to the $D$-mode wave packet. Note that the unit cell changes because the wave packet consists of phonons near the Brillouin zone boundary.

phase of the excited coherent phonons, whose information is inaccessible by use of steady-state and time-resolved Raman measurements.

Graphene with four layers was grown on $\mathrm{SiC} / \mathrm{Si}(001)$ substrate (GOS). The details of this method were reported previously. ${ }^{21}$ We also used defective graphite samples (Ar-G), in which the surface of highly oriented pyrolitic graphite (HOPG) was implanted with a $30-\mathrm{keV} \mathrm{Ar}^{+}$-ion beam to generate local defects. ${ }^{16,22}$ Typical dimensions of the sample were $10 \mathrm{~mm} \times 10 \mathrm{~mm}$. In advance of ultrafast measurements, the $D / G$ ratios of samples were evaluated by conventional Raman spectroscopy with a $532 \mathrm{~nm}$ laser, which resulted in $D / G \sim 1$ for both samples. The grain size of GOS samples, showing high crystallinity of crystalline grains, was estimated to be $10-20 \mathrm{~nm}$ by using the cross-sectional transmission electron microscope. ${ }^{23}$ Nanographite samples with similar grain sizes showed similar $D / G$ ratios ${ }^{24}$ indicating that most of the $D$ mode of the GOS sample originates from the grain boundary (edges). On the other hand, the $D$-mode mostly originates from the point defects induced by the Ar irradiation in the case of Ar-G samples, whose density may be determined by the Ar-ion implantation dose of $3 \times 10^{13} \mathrm{~cm}^{-2} \cdot{ }^{17}$

Figures 2(a) and 2(b) show the obtained reflectivity change as a function of the delay time. The electronic response is subtracted by fitting the data with a single exponential decay, and the low-frequency components are filtered out in the Fourier domain. After the subtractions, high-frequency
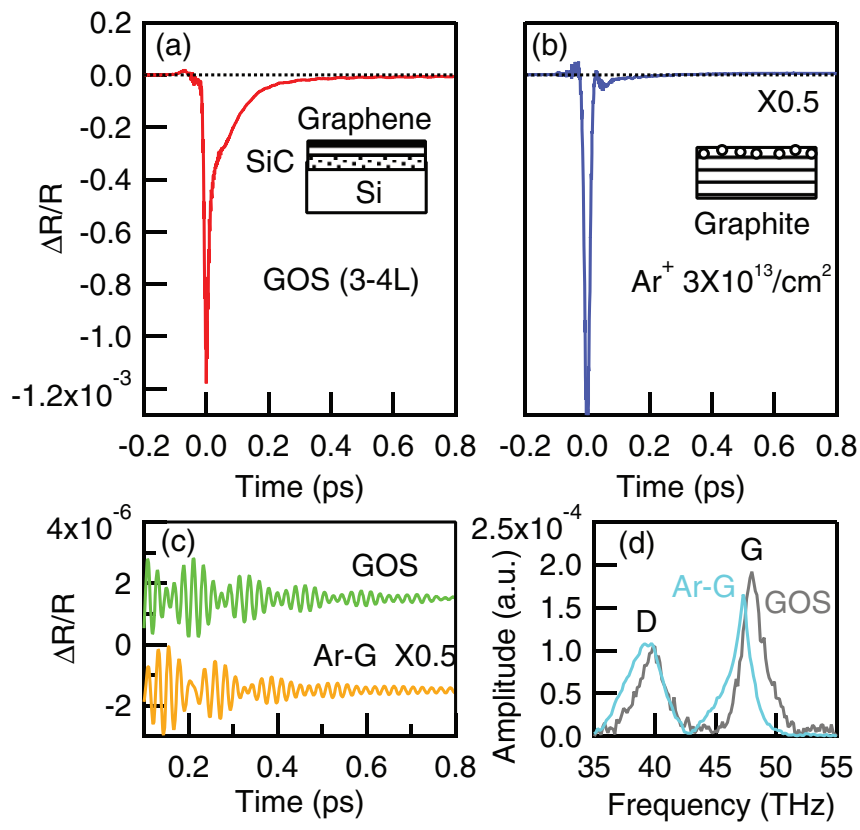

FIG. 2. (Color online) Pump-probe reflectivity measurements. Reflectivity changes in (a) the GOS sample with 3-4 layers of graphene and (b) the $\mathrm{Ar}^{+}$-ion implanted HOPG (Ar-G) with an implantation dose of $3 \times 10^{13}$ ions $/ \mathrm{cm}^{2}$. Schematics of the samples are shown in the insets. (c) Extracted high-frequency coherent phonons in the GOS and Ar-G samples. (d) Fourier spectra of the high-frequency coherent phonons in GOS and Ar-G.

coherent beat oscillations of the $G$ - $(47.4 \mathrm{THz})$ and $D$-mode (40 THz) phonons were observed as shown in Fig. 2(c). The dephasing times of the $G$ and $D$ modes are 0.28 and 0.17 ps for the GOS, and 0.39 and $0.15 \mathrm{ps}$ for the Ar-G, respectively. The slight differences between the $G$ - and $D$-mode frequencies observed in Fig. 2(d) are probably due to the stress-induced high-frequency shift of the $G$ and $D$ modes in GOS and the defect-induced bond softening in Ar-G. ${ }^{12,25}$ Observation of the coherent $D$-mode phonons demonstrates that large-wavevector $D$-mode phonons can be excited simultaneously with fixed phase relations, in the cases of both the edges and the point defects.

The spectral components of the excited large-wave-vector phonon wave packet can be elucidated by resolving the spectrum of the probe light ${ }^{26-28}$ because the wave vector of the coherent $D$-mode phonons $(q \neq 0)$ depends strongly on the detected wavelength due to the double resonance [see Fig. 1(a)]. The obtained FT spectra in Figs. 3(a) and 3(b) of the GOS and Ar-G samples, respectively, show the intensity and frequency dependences of the $D$-mode phonon on the probed wavelength. The rapid increase of the intensity ratio between the $D$ and $G$ modes $(D / G$ ratio) as a function of the probe wavelength [Fig. 3(c)] reflects $\lambda^{4}$ dependence reported in Raman scattering experiments, ${ }^{24}$ which originates from the energy denominators in the doubly resonant Raman scattering cross section. ${ }^{29}$ In our coherent phonon experiment, the stimulated Raman process is used twice in the pump and probe processes in a similar manner to coherent anti-Stokes Raman scattering (CARS), ${ }^{4}$ which yields a $\lambda^{8}$ dependence of the $D / G$ ratio. 

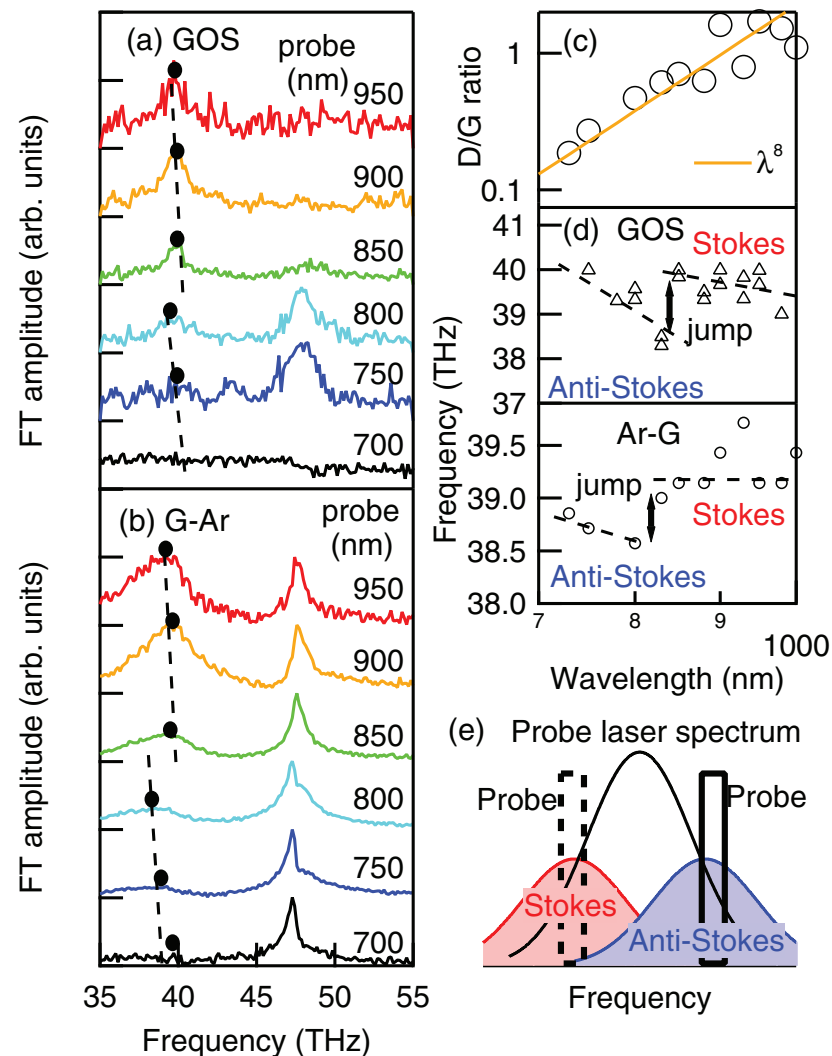

(e) Probe laser spectrum

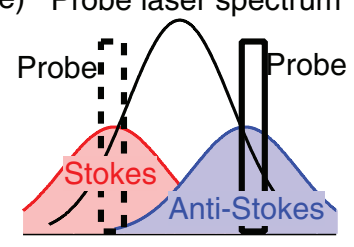

Frequency

FIG. 3. (Color online) The high-frequency coherent phonons. Probe-wavelength dependence of the observed spectra in (a) the GOS sample and (b) the Ar-G with the implantation dose of $10^{13}$ ions $/ \mathrm{cm}^{2}$. The peak frequencies are indicated by circles, and the dashed lines are guides for the eyes. (c) Probe-wavelength dependence and $\lambda^{8}$ dependence (solid line) of the $D / G$ ratio of the coherent phonon amplitudes in the Ar-G. (d) Probe-wavelength dependence of the peak frequency of the GOS and Ar-G. The dashed lines are a guide for the eyes to highlight the frequency jump. (e) Schematic of the Stokes (red shaded area) and anti-Stokes (blue shaded area) signals in the wavelength-resolved coherent phonon experiment.

The probe-wavelength dependence of the $D$-mode frequency [Fig. 3(d)] and the existence of the frequency jump at $\sim 830 \mathrm{~nm}$ demonstrate that many phonons with different wave vectors contribute to the coherent phonon oscillation. The frequency jump can be explained as follows. In the coherent phonon experiment, both the stimulated Stokes and the anti-Stokes processes contribute to the observed signal at a fixed probe wavelength as shown by the red and blue shaded areas in Fig. 3(e). The ratio of the Stokes signal relative to the anti-Stokes signal changes at the center wavelength of the laser. This is illustrated by using the examples of two probe wavelengths, shown by broken and solid areas in Fig. 3(e), in which the Stokes component is dominant in the low-frequency (long wavelength) part of the Gaussian probe spectrum, whereas the anti-Stokes component is dominant in the high-frequency (short wavelength) part of the spectrum. Because in the case of $D$ mode the frequency of the anti-Stokes peak is different from that of the Stokes peak, ${ }^{30}$ the frequency jump appears at the center wavelength of the probe laser, as observed in Fig. 3(d). ${ }^{31}$

One of the interesting phonon properties that appear due to the wave packet is a large time-dependent frequency shift (positive phonon chirp). Normally, frequency chirp is observed through electron softening or stiffening because of the high excitation density of the electrons. ${ }^{11,32,33}$ However, the observed large phonon chirp in the $D$ mode is almost independent of the pump fluence and the chirp direction is the opposite to that of the $G$ mode. ${ }^{11,12,34}$ Therefore, neither decoupling of the Kohn anomaly ${ }^{11}$ nor the electronic softening can explain the main part of the frequency chirp.

To explain the $D$-mode phonon chirp, we first calculate the spectrum of a nanoscale optical-phonon wave packet. As in Fig. 1 , the wave vector of the $D$-mode phonon is determined by the double resonance as

$$
k_{\text {phonon }}=k_{\mathrm{K}}-E_{\text {laser }} / \hbar v_{e},
$$

where $k_{\mathrm{K}}$ and $v_{e}$ are the wave vector at the $K$ point and the Fermi velocity of the Dirac electrons in graphene, respectively. ${ }^{19}$ Considering the spectrum of the laser we used and scattering processes of every resonant Dirac electrons, the spectrum of the $D$-mode coherent phonons $S\left(\omega_{\text {phonon }}\right)$ is estimated as a solid line in the inset of Fig. 4(a). ${ }^{35} S\left(\omega_{\text {phonon }}\right)$ has a broad bandwidth and an asymmetric distribution. ${ }^{35}$ Because we measured the coherent $D$-mode phonons using lower excitation energy $(1.55 \mathrm{eV})$ with the broad spectrum, the estimated $D$-mode phonon spectrum has a lower-lying peak and a broader bandwidth than that calculated for $1.9 \mathrm{eV}$ excitation (broken line). This broad bandwidth is a key to realize nanoscale optical phonon wave packet, and the asymmetry is an important clue to understand the phonon chirp.

The spatiotemporal profile of the $D$-mode phonon $S(t, x)$ is then evaluated as a superposition of excited coherent phonon modes:

$$
S(t, x)=\int d \omega_{\text {phonon }} S\left(\omega_{\text {phonon }}\right) e^{i\left(k_{\text {phonon }}\left(\omega_{\text {phonon }}\right) x-\omega_{\text {phonon }} t\right)} .
$$

We assume that the initial phases of the excited phonons have the same value of 0 because they are impulsively excited by the ultrashort laser pulses, and use the phonon dispersion curve along $\Gamma-K$ direction. $^{8}$ Note that this superposition can only be possible when the phonons are coherently excited. The calculated $D$-mode phonon displacement [Fig. 4(a)] turns out to be a nanoscale wave packet $(\sim 6 \mathrm{~nm})$ because of the broad bandwidth of the excited $D$-mode phonons. Since $D$-mode phonons near the $K$ point interact strongly with Dirac electrons, the wave packet gains a group velocity of $\sim 5 \mathrm{~nm} / \mathrm{ps}$, as indicated by the arrows in Fig. 4(a). This velocity reflects the slope of the phonon dispersion near the $K$ point [Fig. 4(b)] that results from the Kohn anomaly. ${ }^{8}$

$S_{\text {pump }}(t, x)$ enables us to calculate the transient reflectivity $[\Delta R(t) / R]$ due to the $D$ mode with several assumptions. Because the displacement is not homogeneous in the case of $D$-mode phonons, we assumed that observed reflectivity change is proportional to the correlation $\left(S_{\text {pump }} * S_{\text {probe }}\right)$ between the wave packets excited by the pump and probe pulses. Calculated delay-time dependence of the correlation is shown in Fig. 4(c). By performing a time-windowed FT, we can numerically evaluate the chirp profile and the dephasing of the $D$-mode phonons. The calculated phonon chirp in Fig. 4(d) 


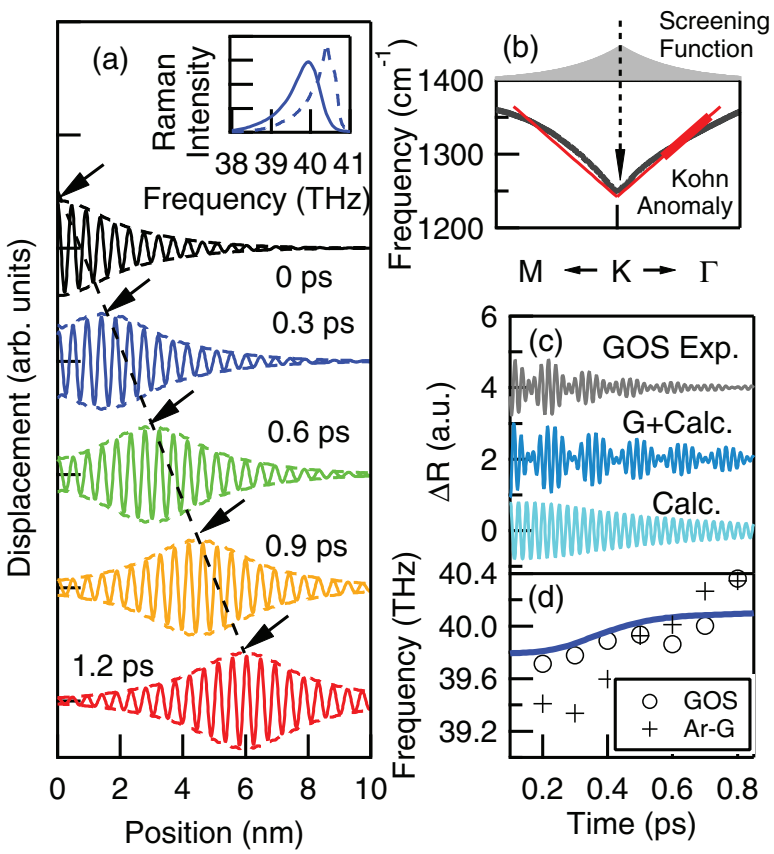

FIG. 4. (Color online) The calculation of the $D$-mode optical phonon wave packets. (a) The wave packet expected to be excited in our experiment. The dashed line and arrows show the propagation of the wave packet. The calculated $D$-mode Raman intensity at $1.9 \mathrm{eV}$ (dashed line $\mathrm{e}^{35}$ ) and that estimated for our coherent phonon experiment (solid line) are shown in the inset. (b) The phonon dispersion curves for the highest optical phonon mode at the $K$ point in graphene. The gray line is taken from Ref. 8, and the red line is the empirical linear dispersion used in the calculation of the wave packet. The thick red line shows the region where phonons are excited in our experiment. The electronic screening function that causes Kohn anomaly is also shown schematically above the plot. (c) The correlation between $S_{\text {pump }}(x, t)$ and $S_{\text {probe }}(x, 0)$, which may be proportional to the coherent phonon signal. Data from the experiment (GOS exp.), calculated result (Calc.), and calculated result summed with the $G$-mode phonon response are shown. (d) Calculated and observed time dependence of the $D$-mode frequency obtained from a windowed Fourier transformation (Hanning window of $0.3 \mathrm{ps}$ ). The solid line is the result of the calculation. The circles and crosses are the experimental data for the GOS, and Ar-G, respectively.

(the solid line) is in qualitatively good agreement with the experimental results especially for the GOS sample.

The calculation predicts the dephasing time of the $D$ mode [0.3 ps as shown in Fig. 4(c)] due to the broad spectral width of
$S\left(\omega_{\text {phonon }}\right)$. This value corresponds to the time required for the wave packet to leave the graphene edge. Assuming that normal dephasing rate neglected in the calculation (coming from anharmonicity, defect or impurity scattering, inhomogeneity, etc.) is the same for both the $G$ and $D$ modes, the difference $\Delta=1 / \tau_{D}-1 / \tau_{G}$ represents the additional dephasing rate of the $D$ mode, where $\tau_{D}$ and $\tau_{G}$ are the dephasing times of the $D$ and $G$ modes, respectively. The experimental values of $1 / \Delta$ ( $0.24 \mathrm{ps}$ for the Ar-G and $0.43 \mathrm{ps}$ for the GOS) are in qualitative agreement with the predicted additional dephasing time for the $D$ mode of $0.3 \mathrm{ps}$. Therefore, all of the observed characteristics of the coherent $D$-mode phonons are consistent with the excitation of nanoscale optical-phonon wave packet, which could open a way to modulate the electronic states or atomic displacements in nanometer-spacial and femtosecond-time scales.

Because the wave vectors of $D$-mode phonons and Dirac electrons in graphene and graphite are similar, the wave packet may open a gap on the nanometer scale ${ }^{36}$ due to the strong electron-phonon coupling. The observed reflectivity change of the $D$ mode may correspond roughly to the amplitude of $8 \mathrm{fm},{ }^{34,37}$ which could open a gap of $4 \mathrm{meV}$ by considering the electron-phonon coupling mechanism. ${ }^{36}$ Note that the $G$ mode just shifts the wave vector of the Dirac point and could not generate a gap owing to the different symmetry of the displaced lattice. ${ }^{36}$ The energy gap does not cancel out until the coherent phonons dephase. Because of this, the Dirac electrons acquire a finite effective mass and slow down. To investigate such an interaction, the transport properties under selective excitation of the coherent $D$-mode phonon could be of interest.

In summary, we have excited a coherent nanoscale opticalphonon wave packet using the impulsive excitation of interDirac-cone scattering at graphene edges and defects. Since the $D$ mode couples strongly with the electronic states near the Fermi level, this wave packet spatiotemporally modulates the electronic states with nanometer-spatial and femtosecondtime resolutions adding finite effective mass to Dirac electrons. Combining the concept proposed here with sophisticated pulse shaping techniques ${ }^{38,39}$ will enable full control of the phases and amplitudes of the optical phonon wave packet, which could be important in order to understand the coherent dynamics of electron-phonon coupling in graphene-related materials.

The authors are very grateful to Prof. Riichiro Saito at Tohoku University for proofreading the manuscript. This work is partially supported by Grant-in-aid for Scientific Research from Japan Society for the Promotion of Science (KAKENHI nos. 23104515, 23104713, 23241034, and 25104712).
*Corresponding authors: jun@ynu.ac.jp; kitajima@1xray.jp

${ }^{1}$ J. L. Harek, W. Wohllenben, R. J. Cogdell, D. Zeidler, and M. Motzkus, Nature (London) 417, 533 (2002).

${ }^{2}$ A. Assion, T. Baumert, M. Bergt, T. Brixner, B. Kiefer, V. Seyfried, M. Strehle, and G. Gerber, Science 282, 919 (1998).

${ }^{3}$ K. W. Kim, A. Pashkin, H. Schäfer, M. Beyer, M. Porer, T. Wolf, C. Bernhard, J. Demsar, R. Huber, and A. Leitenstorfer, Nat. Mater. 11, 497 (2012).

${ }^{4}$ M. Hase, M. Kitajima, A. M. Constantinescu, and H. Petek, Nature (London) 426, 51 (2003).
${ }^{5}$ S. Tomimoto, S. Saito, T. Suemoto, K. Sakata, J. Takeda, and S. Kurita, Phys. Rev. B 60, 7961 (1999).

${ }^{6}$ H. Katsuki, H. Chiba, B. Girard, C. Meier, and K. Ohmori, Science 311, 1589 (2006).

${ }^{7}$ A. K. Geim and K. S. Novoselov, Nat. Mater. 6, 183 (2007).

${ }^{8}$ S. Piscanec, M. Lazzeri, F. Mauri, A. C. Ferrari, and J. Robertson, Phys. Rev. Lett. 93, 185503 (2004).

${ }^{9}$ J. Yan, Y. Zhang, P. Kim, and A. Pinczuk, Phys. Rev. Lett. 98, 166802 (2007). 
${ }^{10}$ C.-F. Chen, C.-H. Park, B. W. Boudouris, J. Horng, B. Geng, C. Girit, A. Zettl, M. F. Crommie, R. A. Segalman, S. G. Louie, and F. Wang, Nature (London) 471, 617 (2011).

${ }^{11}$ K. Ishioka, M. Hase, M. Kitajima, L. Wirtz, A. Rubio, and H. Petek, Phys. Rev. B 77, 121402 (2008).

${ }^{12}$ S. Koga, I. Katayama, S. Abe, H. Fukidome, M. Suemitsu, M. Kitajima, and J. Takeda, Appl. Phys. Exp. 4, 045101 (2011).

${ }^{13}$ K. Kato, K. Ishioka, M. Kitajima, J. Tang, R. Saito, and H. Petek, Nano Lett. 8, 3102 (2008).

${ }^{14}$ J.-H. Kim, K.-J. Han, N.-J. Kim, K.-J. Yee, Y.-S. Lim, G. D. Sanders, C. J. Stanton, L. G. Booshehri, E. H. Hároz, and J. Kono, Phys. Rev. Lett. 102, 037402 (2009).

${ }^{15}$ A. R. T. Nugraha, G. D. Sanders, K. Sato, C. J. Stanton, M. S. Dresselhaus, and R. Saito, Phys. Rev. B 84, 174302 (2011).

${ }^{16}$ K. Nakamura and M. Kitajima, Phys. Rev. B 45, 78 (1992).

${ }^{17}$ B. S. Elman, M. S. Dresselhaus, G. Dresselhaus, E. W. Maby, and H. Mazurek, Phys. Rev. B 24, 1027 (1981).

${ }^{18}$ C. Casiraghi, A. Hartschuh, H. Qian, S. Piscanec, C. Georgi, A. Fasoli, K. S. Novoselov, D. M. Basko, and A. C. Ferrari, Nano Lett. 9, 1433 (2009).

${ }^{19}$ C. Thomsen and S. Reich, Phys. Rev. Lett. 85, 5214 (2000).

${ }^{20}$ R. Saito, A. Jorio, A. G. Souza Filho, G. Dresselhaus, M. S. Dresselhaus, and M. A. Pimenta, Phys. Rev. Lett. 88, 027401 (2001).

${ }^{21}$ H. Fukidome, Y. Miyamoto, H. Handa, E. Saito, and M. Suemitsu, Jpn. J. Appl. Phys. 49, 01AH03 (2010).

${ }^{22}$ A. Jorio, M. M. Lucchese, F. Stavale, and C. A. Achete, Phys. Status Solidi B 246, 2689 (2009).

${ }^{23}$ H. Handa, R. Takahashi, S. Abe, K. Imaizumi, E. Saito, M.-H. Jung, S. Ito, H. Fukidome, and M. Suemitsu, Jpn. J. Appl. Phys. 50, 04DH02 (2011).

${ }^{24}$ L. G. Cançado, K. Takai, T. Enoki, M. Endo, Y. A. Kim, H. Mizusaki, A. Jorio, L. N. Coelho, R. Magalhães-Paniago, and M. A. Pimenta, Appl. Phys. Lett. 88, 163106 (2006).

${ }^{25}$ J. Röhrl, M. Hundhausen, K. V. Emtsev, T. Seyller, R. Graupner, and L. Ley, Appl. Phys. Lett. 92, 201918 (2008).
${ }^{26}$ L. Lüer, C. Gadermaier, J. Crochet, T. Hertel, D. Brida, and G. Lanzani, Phys. Rev. Lett. 102, 127401 (2009).

${ }^{27}$ O. V. Misochko, M. Hase, and M. Kitajima, Phys. Solid State 46, 1741 (2004).

${ }^{28}$ K. Mizoguchi, R. Morishita, and G. Oohata, Phys. Rev. Lett. 110, 077402 (2013)

${ }^{29}$ M. S. Dresselhaus, G. Dresselhaus, R. Saito, and A. Jorio, Phys. Rep. 409, 47 (2005).

${ }^{30}$ V. Zólyomi and J. Kürti, Phys. Rev. B 66, 073418 (2002).

${ }^{31}$ It is known that normally, Stokes and anti-Stokes peaks are the same. However, a $D$ mode is an exception in that the anti-Stokes peak becomes higher than the Stokes peak in frequency. In wavelengthresolved coherent phonon spectroscopy, an inverse process of the Raman measurement is used, and therefore, the Stokes contribution has higher frequency than the anti-Stokes contribution. This is because the anti-Stokes peak in the Raman experiment measures the $\omega+\Omega$ light generated by the laser with frequency $\omega$, whereas the Stokes contribution in wavelength-resolved coherent phonon spectroscopy measures the $\omega$ component that is generated by the spectral component of the pulsed laser at $\omega+\Omega$.

${ }^{32}$ O. V. Misochko, M. Hase, K. Ishioka, and M. Kitajima, Phys. Rev. Lett. 92, 197401 (2004).

${ }^{33}$ H. Takahashi, Y. Kamihara, H. Koguchi, T. Atou, H. Hosono, I. Katayama, J. Takeda, M. Kitajima, and K. G. Nakamura, J. Phys. Soc. Jpn. 80, 013707 (2011).

${ }^{34}$ I. Katayama, S. Koga, K. Shudo, J. Takeda, T. Shimada, A. Kubo, S. Hishita, D. Fujita, and M. Kitajima, Nano Lett. 11, 2648 (2011).

${ }^{35}$ K. Sato, R. Saito, Y. Oyama, J. Jiang, L. G. Cançado, M. A. Pimenta, A. Jorio, G. G. Samsonidze, G. Dresselhaus, and M. S. Dresselhaus, Chem. Phys. Lett. 427, 117 (2006).

${ }^{36}$ G. G. Samsonidze, E. B. Barros, R. Saito, J. Jiang, G. Dresselhaus, and M. S. Dresselhaus, Phys. Rev. B 75, 155420 (2007).

${ }^{37}$ The displacement was estimated from the time-resolved $\mathrm{x}$-ray diffraction data of $\mathrm{Bi}$ instead of graphene, because there are no experiments that directly report the displacement of the highfrequency $\mathrm{C}=\mathrm{C}$ stretching mode. Details of this estimation are briefly described in Ref. 34 .

${ }^{38}$ A. M. Weiner, Ultrafast Optics (Wiley, New York, 2009).

${ }^{39}$ M. W. Wefers and K. A. Nelson, Opt. Lett. 18, 2032 (1993). 